\title{
Carcinosarcoma of the Thoracic Esophagus That Presented as Focal Ulcerative Lesion
}

\author{
Akira Yoneda Takayuki Miyoshi Taiji Hida Hanako Tetsuo \\ Shunsuke Murakami Yoichi Koga Takashi Hamada Amane Kitasato \\ Hiroaki Takeshita Tamotsu Kuroki \\ Department of Surgery, National Hospital Organization Nagasaki Medical Center, Nagasaki, \\ Japan
}

\author{
Keywords \\ Esophageal cancer $\cdot$ Carcinosarcoma $\cdot$ Surgery
}

\begin{abstract}
Esophageal carcinosarcoma is a rare malignant tumor composed of both carcinomatous and sarcomatous elements. We report a case of esophageal carcinosarcoma in a 56-year-old woman with dysphagia. Esophageal ulcerative tumors were detected by endoscopy and resected by thoracoscopic esophagectomy. Carcinosarcoma was confirmed by the presence of both carcinomatous and sarcomatous tumor components. On immunohistochemistry, the sarcomatous area was positive for keratin staining, while the sarcomatous area was positive for vimentin staining. The tumor reportedly had a better prognosis than SCC of the esophagus, especially in terms of survival rate. The patient's disease was classified as ypT3NOMO, ypStage II. No definitive diagnosis was made preoperatively. We report this case along with a review of the literature.
\end{abstract}

(c) 2021 The Author(s).

Published by S. Karger AG, Basel

\section{Introduction}

Carcinosarcomas are rare malignant tumors with both carcinomatous and sarcomatous components [1,2]. Esophageal carcinosarcoma comprises approximately $0.8 \%-2.8 \%$ of all esophageal neoplasms [3]. Based on its histogenesis and biology, this neoplasm has been referred to as spindle cell squamous cell carcinoma (SCC), sarcomatoid carcinoma, or pseudosarcomatous SCC. Esophageal carcinosarcoma often presents at an early stage. It is difficult to establish the standard treatment for esophageal carcinosarcoma due to its low incidence. 


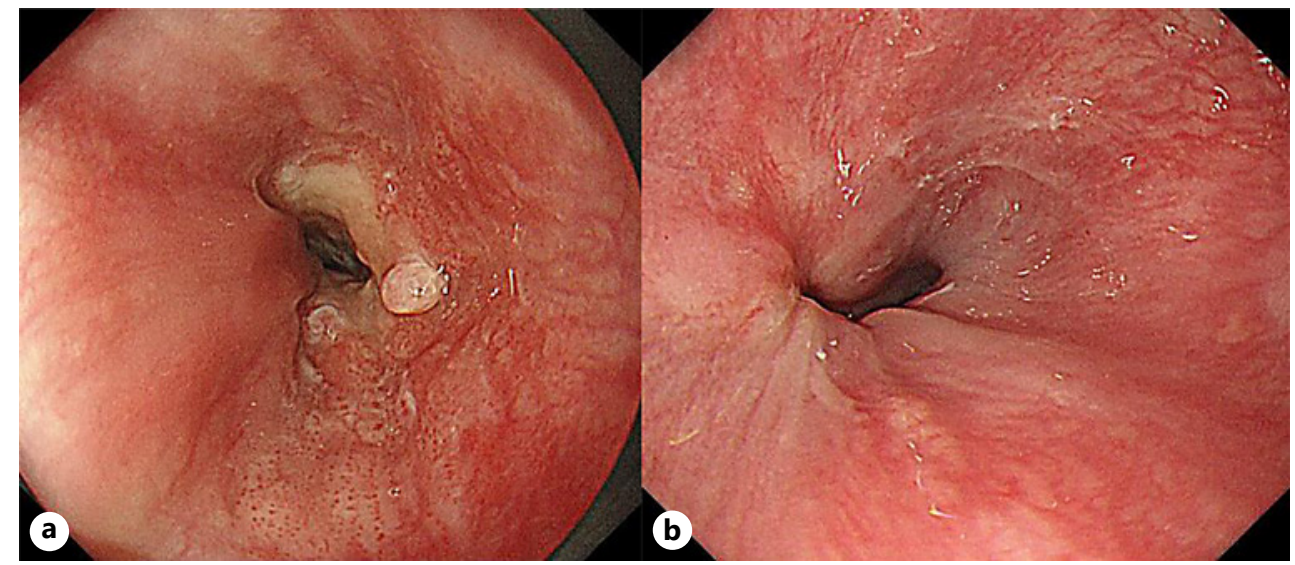

Fig. 1. a EGD examination showed a localized tumor with a $1 / 2$ circumferential ulcer at the lower esophagus to the esophagogastric junction. b EGD examination showed the primary lesion had shrunk and showed scarlike tugging of the ulcer. EGD, esophagogastroduodenoscopy.

Surgical resection is one of the most effective treatments. We present a case of ulcerative esophageal sarcoma in a 56-year-old woman, along with its clinical and immunohistochemical features.

\section{Case Report}

A 56-year-old woman with dysphagia was admitted to our hospital. A barium swallow esophagogram showed a protruding lesion in the lower esophagus. Esophagogastroduodenoscopy (EGD) revealed a localized tumor with a 1/2 circumferential ulcer at the lower esophagus, extending to the esophagogastric junction. A depressed lesion was also found on the oral side, approximately $37 \mathrm{~cm}$ from the incisors (Fig. 1a). On the antral side, gastric flexure invasion was observed. Tumor biopsy revealed SCC. The EGD passed through tumor lesions. No obvious distant metastasis was found. The tumor was diagnosed as cT3N0M0, cStage II, and the patient was treated with 2 courses of preoperative chemotherapy and FP therapy. After chemotherapy, the primary lesion decreased in size and exhibited scar-like tugging of the ulcer (Fig. 1b). The esophagogastric junction wall thickening significantly improved. The findings suggested a partial response according to the Response Evaluation Criteria in Solid Tumors guidelines [4]. Subsequently, thoracoscopic subtotal esophagectomy, 2 regional lymph node dissections, and posterior sternal reconstruction with a gastric conduit were performed. On histopathology, the primary tumor was a $35 \times 25 \mathrm{~mm}$ type 3 tumor with a sarcoma-like growth pattern, consisting of spindle-shaped cells with densely stained enlarged nuclei (Fig. 2). On immunostaining, the spindle-shaped cells were positive for AE1/ AE3 (Fig. 3a) and vimentin (Fig. 3b). They were also partially positive to p40 (Fig. 3c). SCC with scattered, generalized, nuclear, densely stained, swelling, and disturbed polarity was also observed in the stratified squamous epithelium of the ulcer margins. Carcinoma and sarcoma components were observed in the tumor surface and within the tumor, respectively. This led to the diagnosis of esophageal carcinosarcoma. The sarcoma invaded the outer membrane with a depth of ypT3. There was no lymph node metastasis, and the final diagnosis was ypT3ypN0M0 pStage IIA. The patient's response to chemotherapy was grade 1a. Postoperatively, there were no major complications, and the patient was discharged on postoperative day 18. 
Fig. 2. Histopathological findings show sarcoma-like growth pattern consisting of spindle-shaped cells with densely stained enlarged nuclei (original magnification, $\times 100$ ).
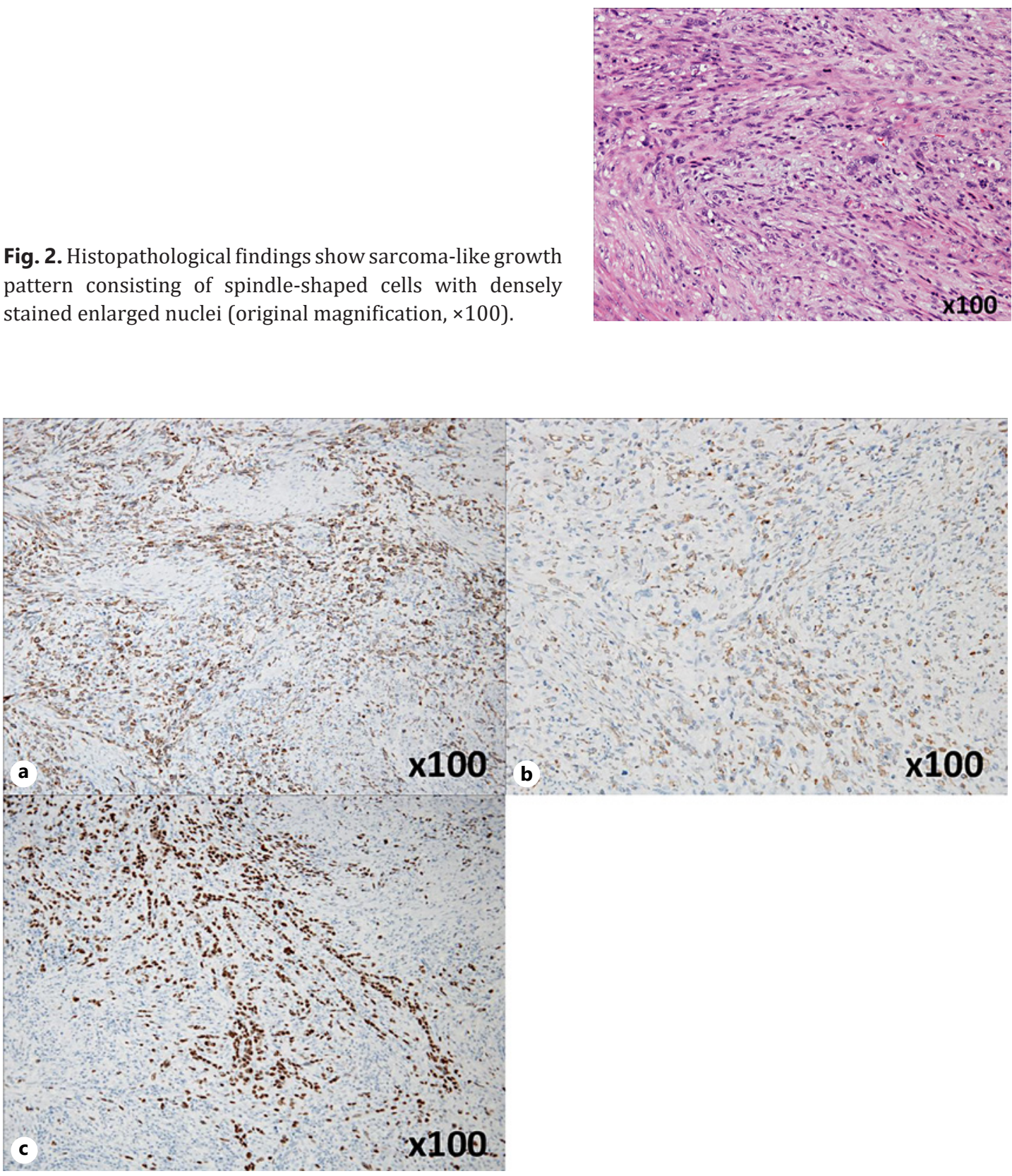

Fig. 3. a Immunostaining of the spindle-shaped cells at the site of growth showed AE1/AE3 positivity (original magnification, $\times 100$ ). $\mathbf{b}$ Immunostaining of the spindle-shaped cells at the site of growth showed vimentin positivity (original magnification, $\times 100$ ). c Immunostaining of the spindle-shaped cells at the site of growth showed partial p40 positivity (original magnification, $\times 100$ ).

\section{Discussion}

Carcinosarcomas are composite malignant tumors with both carcinomatous and sarcomatous components. In Japan, esophageal carcinosarcoma reportedly accounted for $0.1 \%$ $2.8 \%$ of all esophageal tumors [3,5]. Esophageal carcinosarcoma occurs more frequently in middle-aged and older men with a history of smoking, drinking, and SCC of the esophagus [6]. 
Esophageal carcinosarcoma typically presents with a polypoid growth pattern, and the neoplasm does not infiltrate deeply into the esophageal wall. Consequently, patients present with dysphagia and have a favorable survival rate. According to previous reports of esophageal carcinosarcoma, most tumors exhibit a polypoidal morphology. However, our patient was diagnosed with a rare focal ulcerative lesion. Half of the previously reported cases were pT1 $[6,7]$.

Although the etiology of sarcomatous spindle cells remains unclear, this was likely attributed to a monoclonal origin from a single ancestor cell. The sarcomatous components were derived from carcinomatous components via epithelial-mesenchymal transitions or sarcomatous metaplasia [8]. This hypothesis was based on the finding that most carcinosarcoma cases had a transitional zone between the carcinomatous and sarcomatous components, and both elements shared the same genetic alterations [9-11]. Vimentin immunoreactivity indicated the mesenchymal origin of the spindle cells. In the present case, the SCC cells were positive for cytokeratin, while the spindle-shaped tumor cells were immunoreactive to vimentin. Microscopic findings of the transitional zone, true sarcoma components, or both are integral to the diagnosis [12]. Moreover, the application of cytokeratin, an immunohistochemical anti-epithelial marker, helps confirm the presence of the epithelium [13].

The treatment of esophageal carcinosarcoma follows the same protocols for other esophageal cancers. Surgical resection should be considered in patients who can tolerate surgery. The criteria for determining resectability were the same for esophageal SCC and esophageal adenocarcinoma. Given the unpredictable course of esophageal carcinosarcoma, concomitant chemotherapy should be considered to control the microscopic residual disease.

The 5-year survival rate of esophageal carcinosarcoma was not significantly different from that of SCC (27\% vs. $22 \%$ ) [3]. Esophageal carcinosarcoma can cause symptoms earlier than SCC. The rapid growth of this tumor has been previously reported by Sasajima et al. [14]. Sasajima et al. [14] reported a doubling time of 2.2 months for esophageal carcinosarcoma, while other investigators have reported doubling times that were at least twice than that (5 months) of esophageal cancer. Since these tumors are larger than SCC of the same stage, symptoms occur earlier. Patients with esophageal carcinosarcoma have a better chance of survival than patients with typical SCC $[7,15]$. Although the earlier onset of symptoms allows for prompt diagnosis and treatment, the prognosis of esophageal carcinosarcoma is not always favorable.

In esophageal carcinosarcoma, the volume of the sarcoma component is predominant. These sarcomatous cells are more resistant to standard chemotherapy and radiation therapy than the SCC cells. However, no studies have examined the efficacy of chemotherapy or radiation therapy for esophageal and recurrent carcinosarcoma.

Esophageal carcinosarcoma is a rare disease. We report a case of esophageal carcinosarcoma that responded well to preoperative chemotherapy and surgery. Like SCC, chemotherapy and surgical resection improve the long-term survival of patients. More case reports are needed to determine other management strategies for esophageal carcinoma.

\section{Statement of Ethics}

This case report was approved by the Biomedical Ethics Committee. Written informed consent was obtained from the patient for publication of this case report and any accompanying images. This study was approved by the Ethics Committee of the National Hospital Organization Nagasaki Medical Center (Approval Reference No. 2020082).

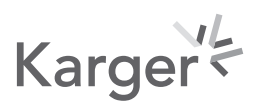




\section{Conflict of Interest Statement}

The authors have no conflicts of interest to declare.

\section{Funding Sources}

There was no financial support.

\section{Author Contributions}

Akira Yoneda, corresponding author, contributed to manuscript writing and literature review. Takayuki Miyoshi contributed to manuscript writing and literature review. Taiji Hida contributed to manuscript writing and literature review. Hanako Tetsuo contributed to manuscript writing and literature review. Shunsuke Murakami contributed to manuscript writing and literature review. Yoichi Koga contributed to manuscript writing and literature review. Takashi Hamada contributed to manuscript writing and literature review. Amane Kitasato contributed to manuscript writing and literature review. Hiroaki Takeshita contributed to manuscript writing and literature review. Tamotsu Kuroki contributed to manuscript writing and literature review. All authors read and approved the final manuscript.

\section{Data Availability Statement}

All data during this study can be directed to the corresponding author.

\section{References}

1 Wick MR. Carcinosarcomas: current perspectives and an historical review of nosological concepts. Semin Diagn Pathol. 1993;10(2):118-27.

2 Sunagawa H, Inamine S, Watanabe M, Kawakami K, Toyama T, Zaha H, et al. Carcinosarcoma of the duodenum: report of a case. Surg Today. 2009;39(10):892-6.

3 Iyomasa S, Kato H, Tachimori Y, Watanabe H, Yamaguchi H, Itabashi M. Carcinosarcoma of the esophagus: a twenty-case study. Jpn J Clin Oncol. 1990;20(1):99-106.

4 Eisenhauer EA, Therasse P, Bogaerts J, Schwartz LH, Sargent D, Ford R, et al. New response evaluation criteria in solid tumours: revised RECIST guideline (version 1.1). Eur J Cancer. 2009;45(2):228-47.

5 Hatch GF 3rd, Wertheimer-Hatch L, Hatch KF, Davis GB, Blanchard DK, Foster RS Jr, et al. Tumors of the esophagus. World J Surg. 2000;24(4):401-11.

6 Kimura H, Konishi K, Kawamura T, Nojima N, Satou T, Maeda K, et al. Esophageal sarcomas: report of three cases. Dig Surg. 1999;16(3):244-7.

7 Wang L, Lin Y, Long H, Liu H, Rao H, He Y, et al. Esophageal carcinosarcoma: a unique entity with better prognosis. Ann Surg Oncol. 2013;20(3):997-1004.

8 Sung CO, Park CK, Kim SH. Classification of epithelial-mesenchymal transition phenotypes in esophageal squamous cell carcinoma is strongly associated with patient prognosis. Mod Pathol. 2011;24(8):1060-8.

9 Amatya VJ, Takeshima Y, Kaneko M, Inai K. Esophageal carcinosarcoma with basaloid squamous carcinoma and rhabdomyosarcoma components with TP53 mutation. Pathol Int. 2004;54(10):803-9.

10 Kashiwabara K, Sano T, Oyama T, Najahima T, Makita F, Hashimoto N, et al. A case of esophageal sarcomatoid carcinoma with molecular evidence of a monoclonal origin. Pathol Res Pract. 2001;197(1):41-6.

11 Matsumoto T, Fujii H, Arakawa A, Yamasaki S, Sonoue H, Hattori K, et al. Loss of heterozygosity analysis shows monoclonal evolution with frequent genetic progression and divergence in esophageal carcinosarcoma. Hum Pathol. 2004;35(3):322-7.

12 Ohtaka M, Kumasaka T, Nobukawa B, Hirai S, Suda K, Ohno Y, et al. Carcinosarcoma of the esophagus characterized by myoepithelial and ductal differentiations. Pathol Int. 2002;52(10):657-63. 
13 Iascone C, Barreca M. Carcinosarcoma and pseudosarcoma of the esophagus: two names, one disease-comprehensive review of the literature. World J Surg. 1999;23(2):153-7.

14 Sasajima K, Taniguchi Y, MorinoYamashita K, Yamashita K, Onda M, Hao K, et al. Rapid growth of a pseudosarcoma of the esophagus. J Clin Gastroenterol. 1988;10(5):533-6.

15 Yoshimoto T, Kobayashi S, Kanetaka K, Kobayashi K, Nagata Y, Morita M, et al. Preoperative chemotherapy with docetaxel, cisplatin, and 5-fluorouracil for locally advanced esophageal carcinosarcoma: a case report and review of the literature. Surg Case Rep. 2018;4(1):18. 